DEPARTMENT OF ENVIRONMENT, TECHNOLOGY AND TECHNOLOGY MANAGEMENT

\title{
The accessibility arc upgrading problem
}

Pablo A. Maya Duque, Sofie Coene, Peter Goos, Kenneth Sörensen \& Fritz Spieksma

\author{
UNIVERSITY OF ANTWERP \\ Faculty of Applied Economics \\ Stadscampus \\ Prinsstraat 13, B.226 \\ BE-2000 Antwerpen \\ Tel. +32 (0)3 2654032 \\ Fax +32(0)3 2654799 \\ http://www.ua.ac.be/tew
}




\title{
FACULTY OF APPLIED ECONOMICS
}

\author{
DEPARTMENT OF ENVIRONMENT, \\ TECHNOLOGY AND TECHNOLOGY MANAGEMENT \\ The accessibility arc upgrading problem
}

Pablo A. Maya Duque, Sofie Coene, Peter Goos, Kenneth Sörensen \& Fritz Spieksma

RESEARCH PAPER 2012-009

MARCH 2012
University of Antwerp, City Campus, Prinsstraat 13, B-2000 Antwerp, Belgium Research Administration - room B.226
phone: (32) 32654032 fax: (32) 32654799
e-mail: joeri.nys@ua.ac.be

The papers can be also found at our website: www.ua.ac.be/tew (research $>$ working papers) \& www.repec.org/ (Research papers in economics - REPEC)

$D / 2012 / 1169 / 009$ 


\title{
The accessibility arc upgrading problem
}

\author{
Pablo A. Maya Duque*1,3, Sofie Coene ${ }^{2}$, Peter Goos ${ }^{1,4}$, Kenneth \\ Sörensen ${ }^{1}$, and Frits Spieksma ${ }^{2}$ \\ ${ }^{1}$ University of Antwerp, Faculty of Applied Economics, ANT/OR \\ ${ }^{2}$ Katholieke Universiteit Leuven, Faculty of Business and Economics, ORSTAT \\ ${ }^{3}$ Universidad de Antioquia, Faculty of Engineering \\ ${ }^{4}$ Erasmus University Rotterdam, Erasmus School of Economics
}

\begin{abstract}
The accessibility arc upgrading problem (AAUP) is a network upgrading problem that arises in real-life decision processes such as rural network planning. In this paper, we propose a linear integer programming formulation and two solution approaches for this problem. The first approach is based on the knapsack problem and uses the knowledge gathered from an analytical study of some special cases of the AAUP. The second approach is a variable neighbourhood search with strategic oscillation. The excellent performance of both approaches is validated using a large set of random generated instances. Finally, we stress the importance of a proper allocation of scarce resources in accessibility improvement.
\end{abstract}

Keywords: Network upgrading problem, knapsack problem, variable neighbourhood search

\section{Introduction}

Accessibility is formally defined by Donnges (2003) as the degree of difficulty people or communities have in accessing locations for satisfying their basic social and economic needs. This concept has been recognised to play an important role in the quality of life as well as the potential for development of communities and regions. The road network is one of the main

\footnotetext{
${ }^{*}$ Corresponding author: Pablo A. Maya Duque, University of Antwerp, Stadscampus S.B.513, Prinsstraat 13, 2000 Antwerp, Belgium, Tel: +3232654061, Fax: +3232654901, Email: pmayaduque@gmail.com
} 
elements that contributes to the accessibility. This is particularly true in rural areas of lesser-developed countries, where the road network ensures the accessibility to the economic and social infrastructure and to facilities, such as hospitals, usually located in regional centres or in more developed cities. In this paper, we study the accessibility arc upgrading problem (AAUP), a network upgrading problem in which resources have to be allocated in order to improve the accessibility to a set of vertices in a network. In the domain of rural road network planning, this problem arises when allocating resources to upgrade roads of a rural transport network, in order to improve the access that communities in small villages have to regional centres. We proceed by giving a precise description of this problem.

The AAUP can be described as follows: Let $\mathcal{G}=(\mathcal{V}, \mathcal{E})$ be a directed connected graph in which the vertex set $\mathcal{V}$ is partitioned into two different sets $\mathcal{V}_{1}$ and $\mathcal{V}_{2}$. Vertices in $\mathcal{V}_{1}$ are called centres, while vertices in $\mathcal{V}_{2}$ are called regular vertices. Each arc $e$ in $\mathcal{E}$ has associated a current level and a set of possible upgrading levels. The level of an arc determines the time required to traverse it. An upgrading cost is incurred when improving an arc from its current level to a specific upgrading level. There is a total budget $B$ to upgrade the level of some arcs. For each vertex $j$ in $\mathcal{V}_{2}$, a weight $w_{j}$ (e.g., number of inhabitants) is given. We define as measure of the accessibility of regular vertex $j$ the travel time from $j$ to the closest centre $i$ in $\mathcal{V}_{1}$. An upgrading strategy specifies a set of arcs to be upgraded and the level to which each of them has to be improved. The objective is to find an upgrading strategy that does not exceed the budget $B$ and minimises the weighted sum of the accessibility measures, i.e., the weighted sum of the times required to travel from each vertex $j$ in $\mathcal{V}_{2}$ to its nearest centre $i$ in $\mathcal{V}_{1}$.

The rest of this paper is structured as follows. In Section 2, we propose a linear integer programming formulation of the AAUP problem. Section 3 reviews the literature, and, in Section 4, we analyse special cases. Section 5 proposes heuristic methods for the AAUP, and, in Section 6, we test these methods on randomly generated instances. Section 7 discusses the potential practical impact of the AAUP. Finally, Section 8 summarises the main contributions of this work and highlights some opportunities for future research on this topic. 


\section{Mathematical formulation}

Based on the mathematical formulation described in Campbell and Lowe (2006), the AAUP can be formulated as a non-linear binary programming model, as shown by Maya Duque and Sörensen (2011). In this paper, we propose an alternative formulation in which the AAUP is defined as a special case of a more general problem called budget constrained minimum cost flow problem (BC-MCFP).

In the BC-MCFP, a given amount of flow has to be sent from a set of supply vertices or sources, through the arcs of a network, to a set of demand vertices or sinks. For each existing arc of the network, there is set of possible upgrading levels. Therefore, for each existing arc, we define one new arc per possible upgrading level connecting the same pair of vertices. For each resulting arc, there is a cost per unit of flow, and a fixed cost associated with the use of the arc. In our particular setting of the BC-MCFP, there is no fixed cost for using an arc at its lowest level, but that cost increases with the upgrading level. The cost per unit of flow decreases as the arc is upgraded. The problem is to find a minimum cost flow, such that the sum of the fixed costs incurred by using some of the arcs at an upgraded level is limited to a fixed budget. Basically, this problem is a minimum cost flow problem that involves an additional set of decision variables related to the upgrading decisions.

Consider the variable $x_{e}$ which is equal to the flow over arc $e$, and a binary variable $y_{e}$ which is equal to 1 if the $\operatorname{arc} e$ is used, and 0 otherwise. Let $\delta^{+}(i)$ and $\delta^{-}(i)$ be the forward and backward stars of vertex $i$, respectively. Furthermore, let parameter $d_{i}$ denote the demand or supply in vertex $i$, and let $p_{e}$ and $c_{e}$ represent the fixed cost of using arc $e$, and the cost per unit of flow over arc $e$, respectively. Note that $d_{i}$ is positive for supply vertices and negative for demand vertices. A formulation for the BC-MCFP is as follows:

$$
\begin{aligned}
& \min \sum_{e \in \mathcal{E}} c_{e} x_{e} \\
& \text { s.t. } \\
& \sum_{e \in \delta^{+}(i)} x_{e}-\sum_{e \in \delta^{-}(i)} x_{e}=d_{i} \\
& \forall i \in \mathcal{V} \\
& x_{e} \leq M y_{e} \\
& \forall e \in \mathcal{E}
\end{aligned}
$$




$$
\begin{array}{lr}
\sum_{e \in \mathcal{E}} p_{e} y_{e} \leq B & \\
\sum_{e: e=(i, j)} y_{e} \leq 1 & \forall i, j \in \mathcal{V}:(i, j) \in \mathcal{E} \\
0 \leq x_{e} \leq a_{e} & \forall e \in \mathcal{E} \\
y_{e} \in\{0,1\} & \forall e \in \mathcal{E}
\end{array}
$$

The objective function (1) minimises the total flow cost. The constraints in (2) ensure that the demand for each sink vertex $j$ is satisfied and that the supply (capacity) for each source $i$ is not exceeded. The constraints in (3), where $M$ denotes a large number, enforce that flow can only pass through arcs that have been selected for use. Constraint (4) imposes an upper bound $B$ on the total upgrading cost. The constraints in (5) ensure that at most one arc connecting each pair of vertices is chosen. Note that these constraints are not needed when the arcs are uncapacitated. Finally, constraints (6) and (7) define the type and bounds for the decision variables. In constraints (6), $a_{e}$ represents the capacity of arc $e$

We now show that the AAUP is a special case of the BC-MCFP. Consider an instance of the AAUP as described in Section 1. Each regular vertex acts as a sink, while each centre is a supply vertex. The value of $d_{j}$ for a regular vertex $j$ is set to $-w_{j}$, while the value of $d_{i}$ for each centre $i$ is set to the total demand on the network (i.e., the sum of the $w_{j}$ values for all $j$ in $\mathcal{V}_{2}$ ). Then, we create one dummy demand vertex connected to each of the centres. The fixed cost and cost per unit of flow for the arcs connecting the dummy vertex and the centres are set to 0 , while the $d_{i}$ value of the dummy vertex is set to $\left(\left|\mathcal{V}_{1}\right|-1\right) \sum_{j \in \mathcal{V}_{2}} w_{j}$. Solving the resulting instance of the BC-MCFP yields a solution for the corresponding instance of the AAUP. Figure 1 shows the transformation of an AAUP into a BC-MCFP, schematically.

In Figure 1, the vertices $c_{1}, c_{2}$ and $c_{3}$ are the centres, while the vertices 1 to 19 represent the regular vertices. The solid lines correspond to existing arcs of the network, while the dashed lines are possible upgrading levels of the existing arcs. The vertex labelled with an asterisk represents a dummy regular vertex and the grey dotted lines are the arcs that connect the dummy vertex to the centres. 


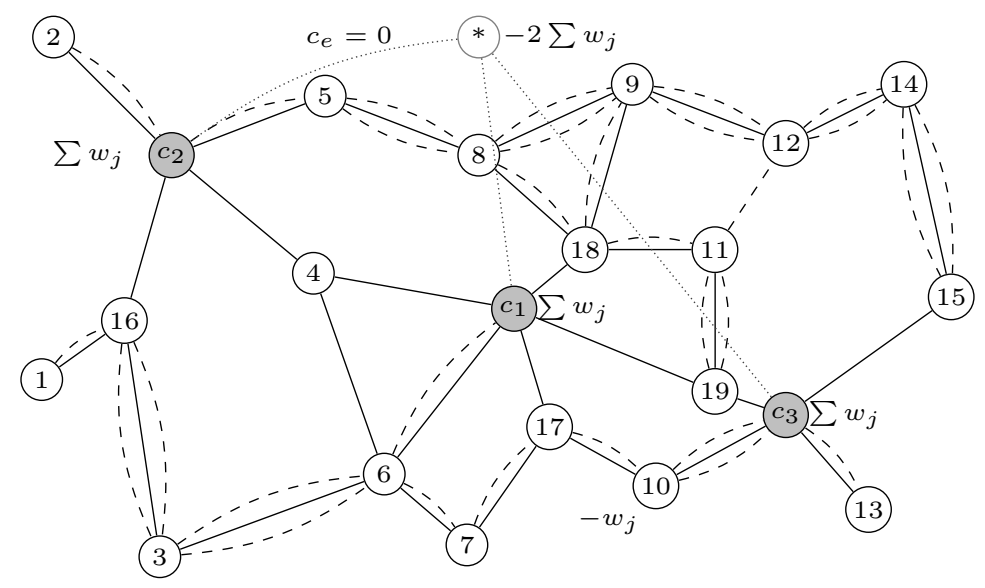

Figure 1: Transformation of the AAUP into a BC-MCFP

\section{Literature review}

In this section, we review the literature that is relevant for the accessibility arc upgrading problem. We first concentrate on the network upgrading problem. Afterwards, we extend the review to consider the accessibility factor within the upgrading network problem.

Although several authors have addressed network upgrading problems, the literature is not as extensive as it is for other problems within the domain of network design. Krumke et al. (1998) distinguish two kinds of upgrading problems depending on whether the focus is on upgrading the arcs or upgrading the vertices. The authors propose a bi-objective approach for both types of problems. In that approach, a sub-class of graphs is $\mathcal{S}$ considered (e.g., the set of spanning trees) and a budget or target value is defined for the first objective. The goal is to find a network within the fixed budget that belongs to $\mathcal{S}$ and minimises the second objective. Results on the complexity of a number of node-based and edge-based upgrading problems are presented. In particular, the case in which the objectives are defined as minimising the cost of improving the network and minimising the total length of the minimum spanning tree is shown to be NP-hard for trees and general networks. Drangmeister et al. (1998) study a related problem that looks for an optimal reduction strategy (i.e., shortening some of the edges) such that a budget constraint is satisfied and the total length of a minimum spanning tree in the modified network is minimised. Some NP-hardness results, even 
for simple classes of graphs, are presented, as well as some approximation algorithms.

Campbell and Lowe (2006) address two $q$-upgrading arc problems that involve finding the best $q$ arcs to upgrade in a network. The $q$-upgrading arc diameter problem requires finding $q$ arcs to upgrade such that the travel time on the maximum shortest path between any origin-destination pair (i.e., the diameter of the network) is minimised. The $q$-upgrading arc radius problem requires finding $q$ arcs to upgrade and locating the vertex center, i.e., the node for which the maximum shortest path to the other nodes in the network (i.e., the radius of the network) is minimised. The two problems are shown to be NP-hard on general graphs, but polynomially solvable on trees. A variant of the problems, which involves a budget constraint, is also studied. It is shown that these problems are NP-hard for general graphs and even for a path graph. Three heuristic algorithms are proposed to deal with these kinds of problems.

Accessibility maximization has been considered mainly in the domain of road network planning. Antunes et al. (2003) consider a inter-urban road network long-term planning problem. They propose a non-linear combinatorial optimization model that does not involve an evolution of the network over time but defines the final status of the network at the end of the planning horizon. Two different heuristics approaches to solve the model are described, based on local search and simulated annealing principles. Santos et al. (2010) extend the study to consider accessibility and robustness objectives simultaneously. A model to help road authorities in their strategic, system-wide decisions regarding the long-term evolution of an interurban road network is proposed. Scaparra and Church (2005) also tackle a road network planning problem, but focus on the rural case for developing countries. The authors propose a GRASP (Greedy Randomized Adaptive Search Procedure) and path relinking heuristic, and consider a bi-objective model, which minimizes the sum of the weighted shortest paths between all pairs of nodes and maximizes the traffic flow. The maximal covering network improvement problem is studied by Murawski and Church (2009) with the objective of improving accessibility to rural health service. The problem is formulated as an integer linear programming problem, and is applied to a real case in the Suhum District of Ghana. The convenience of using a metaheuristic approach for larger instances is pointed out by the authors. 


\section{Analytical analysis}

We study the AAUP for two specific network topologies and present some basic results on the complexity of this problem. First, in Subsection 4.1, we consider the AAUP for the case in which the network topology is a star. Second, in Subsection 4.2, we study the case in which the network is a tree. Throughout this analysis, we denote by AAUP $/ p / q / \mathcal{S}$ the AAUP problem that considers at most $p$ upgrading levels for each arc, exactly $q$ centres, and a network topology as indicated by $\mathcal{S}$. Additionally, we define $s^{l}(e)$ as the reduction in the cost per unit of flow when the arc $e$ is upgraded to level $l$, compared to the cost when the arc is at its original level.

\subsection{AAUP on star network topologies}

Consider first the case in which there is only one centre $c$, one possible upgrading level for each arc, and the network has a star topology centred in $c$. We denote this problem as AAUP $/ 1 / 1 /$ star. Define the binary variable $x_{e}$ to indicate whether arc $e$ is selected for upgrading $\left(x_{e}=1\right)$ or not $\left(x_{e}=0\right)$. Additionally, for each arc $e$, we define a benefit $b_{e}$ and a cost $p_{e}$. The benefit $b_{e}$ is defined as the product of $s^{1}(e)$ and the weight $w_{j}$ of the vertex $j$ that is connected to the centre by means of arc $e$. The cost $p_{e}$ corresponds to the cost of upgrading arc $e$. Then, the AAUP can be reformulated as the following knapsack problem:

$$
\begin{aligned}
\max & \sum_{e \in \mathcal{E}} b_{e} x_{e} \\
& \sum_{e \in \mathcal{E}} p_{e} x_{e} \leq B \\
& x_{e} \in\{0,1\}
\end{aligned}
$$

Thus, the AAUP/1/1/star can be transformed into a knapsack problem and each instance of the knapsack problem can be transformed into an instance of the AAUP $/ 1 / 1 /$ star. Therefore, the two problems are polynomially equivalent, and, in particular, the AAUP/1/1/star is at least as hard as the knapsack problem. In other words, the AAUP/1/1/star is NP-hard.

For the more general case in which $q$ centres are considered and each arc might have more than one upgrading level, AAUP $/ p / q /$ star, the star topology looks slightly different. While each arc still connects a centre and a regular vertex, a regular vertex may now be adjacent to multiple centres. 
An example of this case is presented in Figure 2, where, again, $c_{1}, c_{2}$ and $c_{3}$ are centres, the vertices 1 to 13 represent the regular vertices, the solid lines correspond to existing arcs of the network, and the dashed lines are possible upgrading levels of the existing arcs.

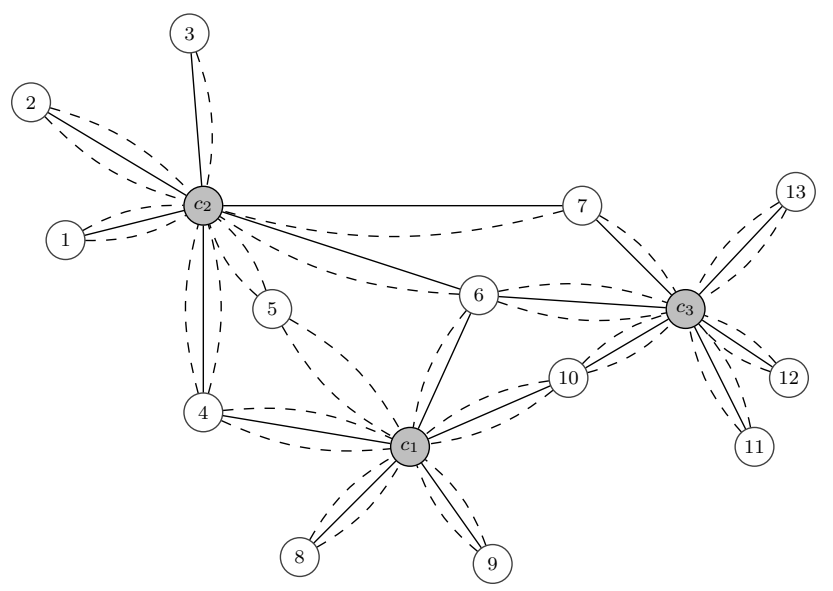

Figure 2: Example of a graph induced by an AAUP with a star topology

By following an analysis similar to the one we did for the AAUP $/ 1 / 1 /$ star, the AAUP $/ p / q /$ star can be formulated as the multi-choice knapsack problem (MCKP) presented in (11)-(14). In this formulation, $\mathcal{E}(j)$ is the set of arcs adjacent to vertex $j, \mathcal{L}_{e}$ is the set of possible upgrading levels for arc $e, x_{e l}$ is a binary variable that indicates whether arc $e$ is improved from its current level to level $l$, and $p_{e l}$ and $b_{e l}$ are the corresponding fixed cost and benefit, respectively. The MCKP has been shown to be NP-hard (Kellerer et al. (2005)).

$$
\begin{array}{rr}
\max & \sum_{e \in \mathcal{E}} \sum_{l \in L_{e}} b_{e l} x_{e l} \\
& \sum_{e \in \mathcal{E}} \sum_{l \in \mathcal{L}_{e}} p_{e l} x_{e l} \leq B \\
\sum_{e \in \mathcal{E}(j)} \sum_{l \in \mathcal{L}_{e}} x_{e l} \leq 1 & \forall j \in \mathcal{V}_{2} \\
x_{e l} \in\{0,1\} & \forall e \in \mathcal{E}, \forall l \in \mathcal{L}_{e}
\end{array}
$$




\subsection{AAUP on tree network topologies}

We study now the case in which the network is a tree. Initially, we consider the problem with only one centre and one possible upgrading level for each arc, AAUP $/ 1 / 1 /$ tree. This problem can be polynomially reduced to the case in which the network is a star. Therefore, the AAUP $/ 1 / 1 /$ tree problem is as hard to solve as the knapsack problem. As a result, it is also NP-hard.

In order to reduce an AAUP/1/1/tree to the problem on a star, we consider, without loss of generality, the tree rooted at the vertex corresponding to the centre. For each regular vertex $j$, we define a cumulative weight $\widehat{w}_{j}$. This cumulative weight is the result of adding $w_{j}$ and the weights $w_{k}$ for all the vertices located after $j$ when the tree is traversed from the root to the leaves. Additionally, for each arc $e$, there is an upgrading cost $p_{e}$, and a benefit $b_{e}=s^{1}(e) \cdot \widehat{w}_{j}$, where $j$ is the arc's vertex that is furthest from the root. The problem can then be formulated as a knapsack problem using the model described in (8)-(10).

Consider now the case in which there are exactly two centres, $c_{1}$ and $c_{2}$. Under this assumption, the set of vertices can be divided into three groups. The first group is formed by $c_{1}$ and all vertices for which the path to $c_{2}$ passes through $c_{1}$. Similarly, the second group consists of by $c_{2}$ and the vertices whose path to $c_{1}$ passes through $c_{2}$. The vertices for which both the path to $c_{1}$ does not pass by $c_{2}$ and the path to $c_{2}$ does not pass through $c_{1}$ form the third group. An example of the AAUP/1/2/tree case is presented in Figure 3. In this picture, the vertices $c_{1}$ and $c_{2}$ are the centres and vertices 1 to 9 represent regular vertices, while the numbers next to the arcs define the time required to traverse the arc at the given status. For this particular example, the set of vertices is partitioned into three groups. The first group is formed by vertices $\left\{c_{1}, 1,2,3\right\}$, while the second group is $\left\{c_{2}, 7,8,9\right\}$, and vertices $\{4,5,6\}$ form the third group.

For any solution, regardless of which arcs are upgraded, $c_{1}$ will be the closest centre for the vertices in the first group and $c_{2}$ will be the closest centre for the vertices in the second group. For the vertices in the third group, which centre is the closest may depend on the upgrading decisions. However, note that this third group can always be partitioned into two different sets of connected vertices. One of those sets will have $c_{1}$ as its closest centre, while $\mathrm{c}_{2}$ will be the closest centre for the other set. The frontier that separates those two sets is always one of the arcs that form the shortest path between 


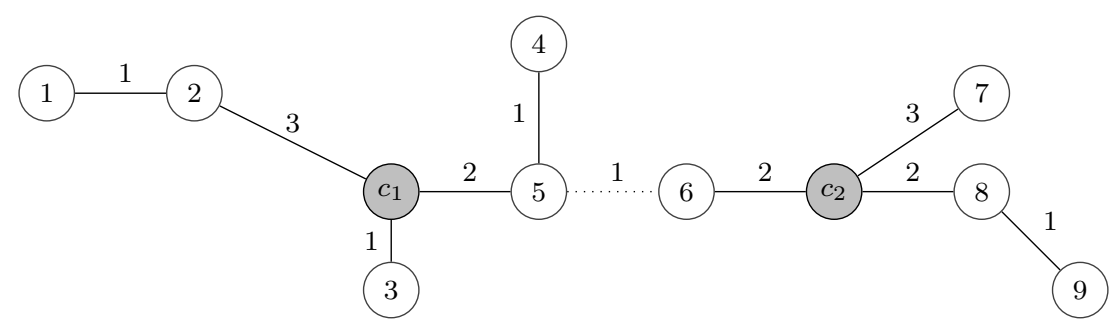

Figure 3: Example of an AAUP/1/2/tree problem

$c_{1}$ and $c_{2}$. For example, in Figure 3, vertices $\{4,5\}$ have $c_{1}$ as their closest centre, while $c_{2}$ is the closest centre for vertex $\{6\}$. The arc $(5,6)$, depicted using a dotted line, is the frontier between those two sets.

When an arc $r$ in the shortest path between the two centres is removed (e.g., arc $(5,6))$, the original graph is decomposed into two trees $\mathcal{T}_{a}=\left\{\mathcal{V}_{a}, \mathcal{E}_{a}\right\}$ and $\mathcal{T}_{b}=\left\{\mathcal{V}_{b}, \mathcal{E}_{b}\right\}$. As we previously showed, each of these trees can be transformed into a star. Therefore, finding the optimal upgrading strategy for the case involving two centres requires the solution of the problem on two stars, which can be integrated into a single multi-choice knapsack problem. By the definition of a tree, there can be at most $n-1 \operatorname{arcs}$ in the shortest path between the two centres. As a result, there are at most $n-1$ options for choosing the arc $r$ to be removed. Thus, an optimal solution for the AAUP $/ 1 / 2 /$ tree can be found by solving $\mathcal{O}(n)$ (multi-choice) knapsack problems and choosing the best out of the $n-1$ solutions.

Using a similar analysis, it is possible to show that, for the general case, in which there are $q$ centres and each arc might have more than one upgrading level, an optimal solution for the AAUP on trees can be found by solving at most $\mathcal{O}\left(n^{q-1}\right)$ MCKPs and choosing the best solution among them. By removing $q-1$ arcs, one in each shortest path that connects two consecutive centres, the tree can be decomposed into $q$ independent sub-trees. The arc upgrading problem on each of those sub-trees can be reformulated as a MCKP by considering an approach similar to the one used for the AAUP $/ 1 / 1 /$ tree. As there are at most $n-(q-1)$ arcs connecting two centres, there are at most $(n-(q-1))^{q-1}$ possibilities for removing the $q-1$ arcs, that is, for defining the set of sub-trees. 


\section{Solution methods}

In Section 2, we have described a formulation that can be used to solve instances of the AAUP using a dedicated MIP solver. In this section, we propose two alternative approaches. First, we describe a heuristic approach specifically designed for the AAUP that is based on the analytical analysis we have done in the previous section. Second, we outline a variable neighbourhood search approach (Hansen and Mladenovic, 2005) for the general BC-MCFP, and apply it to the AAUP, which, as we explained in Section 2, is a special case of the BC-MCFP

\subsection{A knapsack problem-based heuristic for the AAUP}

We have shown that, for the cases in which the network is a star or a tree, the AAUP can be tackled by transforming it into either a knapsack problem or a multi-choice knapsack problem. Based on these basic cases, we outline an approach for the AAUP on general networks. In order to do that, note that, for any feasible solution of the AAUP, we can assign each vertex to its nearest centre. The paths linking each centre to the vertices assigned to it define a forest composed of $\left|\mathcal{V}_{1}\right|$ trees. Therefore, an (exponential time) exact algorithm for the AAUP enumerates all the forest sub-graphs that can be defined from the original graph and in which each tree of the forest is rooted in a different centre. Then, the problem on each of these forests is solved by the approach we describe in Section 4.2. The best among all those solutions corresponds to the optimal solution for the AAUP.

Clearly, from a practical point of view, this approach is not feasible for realistically sized instances. However, by using some key ideas from that exact algorithm, we have built a heuristic for the AAUP, which we refer to as the knapsack problem-based heuristic. In that heuristic, we first create an initial solution. This solution specifies a particular status of the network in which each arc is fixed at a given upgrading level. Based on that particular network, each vertex is assigned to its closest centre by using the minimum cost flow formulation of the AAUP. The paths linking each regular vertex to its closest centre form a forest involving only a subset of the arcs of the complete graph. Then, the arc upgrading problem is solved on that forest. We call this sub-problem the restricted AAUP, and solve it by transforming it into a multi-choice knapsack problem (see Section 4). The resulting solution is a feasible solution for the original AAUP. In that solution, the arcs that are not part of the forest are set to their original level, while the upgrading 
level of the other arcs is dictated by the solution of the restricted AAUP. Based on this new solution, a new assignment of the regular vertices to the centres is made and the entire procedure is iterated. The algorithm stops when a local optimum is reached, i.e., when there is no improvement after iterating over a given feasible solution. Algorithm 1 presents a schematic overview of the KP-based heuristic.

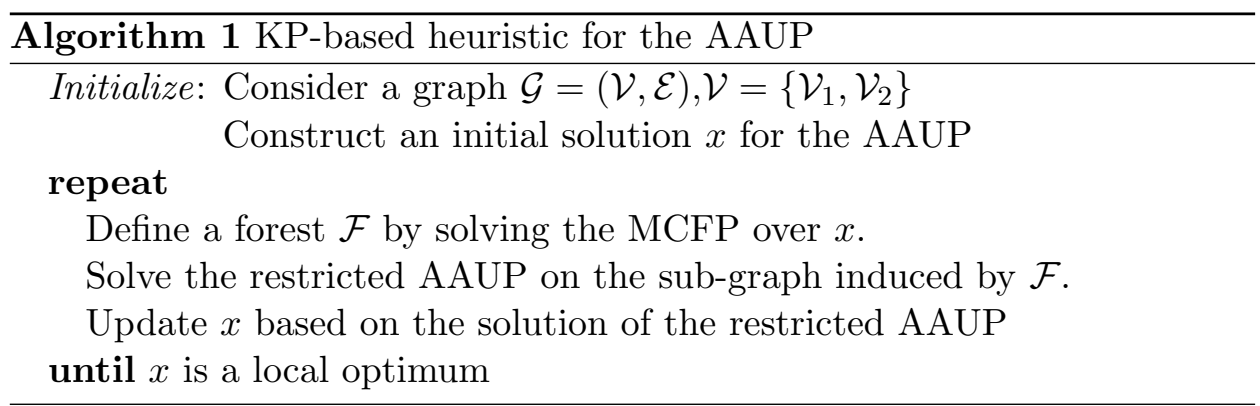

The solution obtained by the KP-based heuristic proposed in Algorithm 1 depends on the initial solution $x$. We consider three different initial solutions: (i) The present solution in which the level of each arc is set to its original (lowest) status; (ii) The ideal solution in which the arcs are assigned the best possible level; and (iii) a random feasible solution in which the level of each arc is set randomly. The algorithm is run once for the first two initial solutions while a given number of replications are performed using different initial random solutions.

\subsection{Variable neighbourhood search for the AAUP.}

In this section, we describe a metaheuristic approach developed for the BC-MCFP and that we have used to solve instances of the AAUP. The approach is a variable neighbourhood search (VNS) with strategic oscillation that considers a feasible upgrading strategy (i.e., a particular status of the network in which each arc is fixed at a given upgrading level) at each iteration. Given an upgrading strategy, the accessibility value in the objective function of the AAUP can be obtained by solving a standard minimum cost flow problem. Two basic moves, upgrade and downgrade, are used to define two neighbourhoods. The first neighbourhood $\left(\mathcal{N}_{1}\right)$ contains all solutions that can be reached from the current solution by upgrading the level of an arc. The second neighbourhood $\left(\mathcal{N}_{2}\right)$ includes all the solutions that can be obtained from the current solution by considering both moves simultane- 
ously. Whenever the search reaches a local optimum, a strategic oscillation is applied. This oscillation allows the search to temporarily consider infeasible solutions and restarts the VNS once the feasibility has been restored. Additionally, a shaking phase is applied when the strategic oscillation fails to move the search to a better solution. Algorithm 2 presents an outline of the VNS approach.

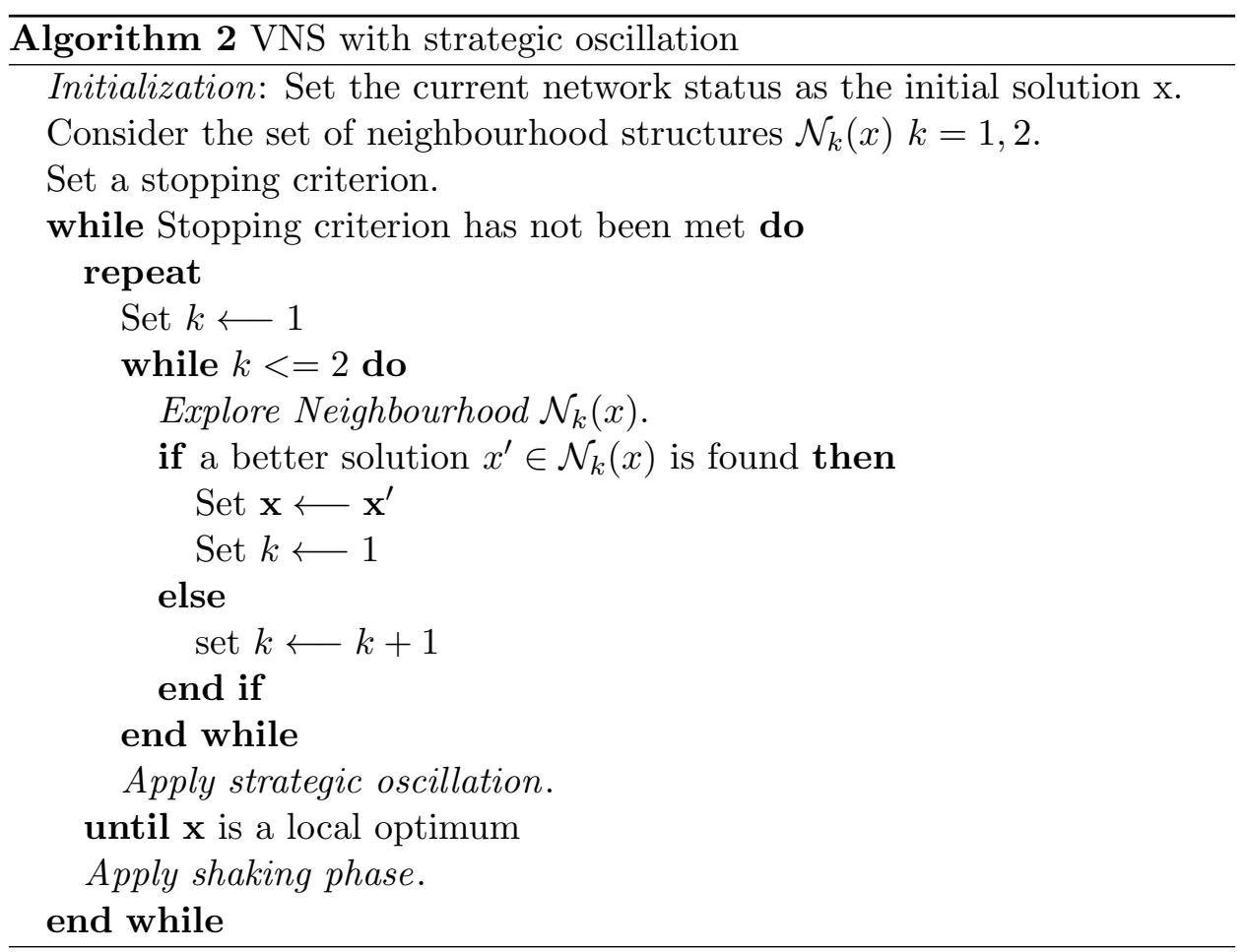

\subsubsection{Upgrading move}

The upgrading neighbourhood $\mathcal{N}_{1}$ is formed by all the solutions that can be reached from the current solution by upgrading an arc by one level without violating any constraint. In order to estimate the cost-saving potential of an arc whose upgrading is feasible, we use the solution of the MCFP associated to the current solution to distinguish between basic and non-basic arcs. Basic arcs are those for which the associated decision variable is basic when solving the linear problem, while non-basic arcs are related to the non-basic decision variables. For the basic arcs, the saving is estimated as the product of the reduction in cost per unit of flow obtained by upgrading the arc 
and the flow that passes the arc in the current solution. The saving for a non-basic arc is estimated by its reduced cost. The arc to be upgraded is selected randomly among the $\alpha$ arcs that provide the best saving estimates.

After upgrading an arc, a procedure to remove redundant upgraded arcs is run. That procedure downgrades to their lowest level all the arcs that have been upgraded in previous iterations of the VNS algorithm but do not carry any flow in the current solution. We use the solution of the MCFP associated to the current upgrading strategy to identify the arcs that must be downgraded. The solution obtained by downgrading those arcs will have the same objective value but it will use less resources.

\subsubsection{Combined upgrading-downgrading move}

The neighbourhood $\mathcal{N}_{2}$ contains all feasible solutions that can be obtained from the current solution by downgrading one arc and upgrading at least one other arc. This neighbourhood requires a list of upgrading candidates and a list of downgrading candidates. The former list is formed by all the arcs that are basic in the current solution and can be upgraded. A saving cost is estimated for each of those arcs, in the way described for the upgrading move in Section 5.2.1. That saving is used to order the upgrading candidates. The list of downgrading candidates contains all the arcs that can be downgraded, i.e., the arcs for which the current level is not the lowest. This list is arranged in decreasing order of the ratio of the fixed cost for using the arc at its current level to the flow through the arc in the current solution. Only the $\beta$ best candidates in this list are kept.

For every downgrading candidate, the upgrading list is traversed in decreasing order of saving potential searching for a feasible upgrading candidate. Whenever a feasible candidate is found, the remainder of the list is traversed in order to add as many upgrading candidates as possible. As a result, whenever a feasible move is identified, it involves one arc to be downgraded and either one arc or a set of arcs to be upgraded. Each of the feasible moves is evaluated by solving a minimum cost flow problem. In order to limit the computational effort, a first improvement approach is used, i.e., the search is stopped as soon as a feasible move is found that improves current solution. 


\subsubsection{Strategic oscillation and shaking}

The strategic oscillation component aims to help the search escape from local optima and explore different parts of the solution space. This is done by allowing the search to temporarily consider infeasible solutions by relaxing the budget constraint. Based on the solution of the MCFP associated with the current solution, we select arcs to be upgraded ignoring the budget constraints. To that end, the arcs are grouped in two different lists depending on whether they are basic or not. A saving is estimated for each arc in the same way it was described for the upgrading move in Section 5.2.1, and the arcs are ranked in decreasing order of the savings. Then, the best candidate in each list is upgraded. This is repeated as long as the total upgrading cost does not exceed $\gamma$ times the original budget, where $\gamma$ is a user-specified tuning parameter.

After this upgrade, the MCFP corresponding to the new upgrading strategy is solved and its solution is used to run a procedure to restore feasibility. That procedure calculates for all arcs the ratio of the fixed cost for using them at their current level to the flow that passes through them in the current solution. The arcs with the largest ratio are downgraded to their lowest level until the budget feasibility is restored.

When the strategic oscillation fails to find a better feasible solution, a shaking phase is applied to restart the search from a significantly different solution. This phase downgrades all the arcs that carry flow in the current solution to the lowest level, while, for arcs that do not carry flow, the level is set randomly. After ensuring that this new solution is feasible, the search is restarted, giving rise to a new iteration of the VNS. The number of iterations of the shaking phase is used as a stopping criterion for the VNS algorithm.

\section{Computational Results}

In this section, we evaluate the performance of the two heuristic solution approaches based on a set of 480 randomly generated instances of the problem. The mathematical model and the two algorithms for solving the AAUP were implemented in Java and ILOG CPLEX Concert Technology (IBM ILOG CPLEX Optimization Studio Academic Research Edition V12.2). 


\subsection{Instance generation}

To generate the 480 AAUP instances, we first created a set of 30 random instances for the minimum cost flow problem using GNETGEN, which is a modification of the widely used NETGEN generator proposed by Klingman et al. (1974). Table 1 shows the main parameters that were used for the generator. The numbers of sources (centres), transhipments and sinks (regular vertices) are expressed as percentages of the total number of vertices $n$. The number of arcs is defined as a percentage of the number of arcs in a complete graph. Finally, the total supply was set to 100 times the number of vertices.

Table 1: Parameters used for generating random MCFP instances

\begin{tabular}{lc}
\hline Parameter & Values \\
\hline Number of vertices $(n)$ & $100,200,400,500,1000$ \\
Percentage of sources $(\%)$ & 2,5 \\
Percentage of transhipments $(\%)$ & 30 \\
Percentage of arcs $(\%)$ & $3,5,15$ \\
Total supply/demand & $100 n$ \\
Minimum cost for arcs & 1 \\
Maximum cost for arcs & 100 \\
\hline
\end{tabular}

These minimum flow cost networks form the starting point to generate the AAUP instances. In those instances, $\mathcal{V}_{1}$ corresponds to the sources or supply vertices, the set $\mathcal{V}_{2}$ contains all sinks, and, for each vertex $j$ in $\mathcal{V}_{2}$, the demand represents the weight $w_{j}$. For each arc of the original network, we generated a set of upgrading options or copies. In order to make the set of instances as diverse as possible, we use two different procedures to generate these copies: the number of copies for each arc is either a fixed number $m$ or a random number between 1 and $m$. We used two values for $m$, namely 2 and 3. Each copy of an arc has a cost per unit of flow and a fixed cost for using the arc. The flow cost decreases with each extra copy of an arc, while the fixed cost increases. For each arc, the cost per unit of flow at the lowest level (copy 0 of the arc) is the cost generated by GNETGEN, while the fixed cost for using that arc is 0 . For the $r$-th copy of an $\operatorname{arc} e$, the flow cost $c_{e}^{r}$ and fixed cost $p_{e}^{r}$ were generated using

$$
c_{e}^{r}=c_{e}^{r-1} / 2+U\left(0, c_{e}^{r-1} / 2\right)
$$


and

$$
p_{e}^{r}=p_{e}^{r-1}+U\left(0, c_{e}^{0}\right)
$$

where $U(a, b)$ represents a continuous uniformly distributed random variable on the interval $[a, b]$.

Finally, four different budget values are considered for each AAUP network. To compute these budget values, we first solved the minimum cost flow problem over the original network, i.e., the network in which all arcs are set at their lowest level. Then, the total budget was defined as the sum of the cost of upgrading all the arcs that carry flow in the optimal solution to their best possible level. The four different budget values correspond to four different percentages of this total budget. In total, 480 instances were generated as a result of combining the 30 random minimum cost flow networks and the parameter values described in the Table 2 .

Table 2: Experimental parameters for generating the AAUP instances

\begin{tabular}{lc}
\hline Parameter & Values \\
\hline Copy Procedure & Fixed, Random \\
Number of Copies $(m)$ & 2,3 \\
Budget values $(\%)$ & $20,50,70,100$ \\
\hline
\end{tabular}

We used Cplex to solve the AAUP instances using the mathematical model described in Section 2 with a time limit of one hour. Table 3 shows the number of times the optimal solution was found within the time limit for the 24 instances at each combination of number of vertices and budget. The results show that it becomes harder to solve the instances to optimality when their size, as measured by the number of vertices, increases. The hardest type of problem is one involving a larger number of vertices and a small budget. For the instances for which the optimum was not found, the average gap to optimality was $2.3 \%$, with a maximum of $30.1 \%$.

\subsection{Results for the KP-based heuristic}

We first study the KP-based heuristic for the AAUP, as described in Section 5.1. As pointed out, three different kinds of initial solutions were considered: (i) one based on the present status of the network, (ii) one based on 
Table 3: Number of instances solved to optimality using the mathematical model

\begin{tabular}{cccccc}
\hline & \multicolumn{5}{c}{ Number of vertices } \\
\cline { 2 - 6 } Budget (\%) & 100 & 200 & 400 & 500 & 1000 \\
\hline 20 & 23 & 19 & 11 & 6 & 2 \\
50 & 23 & 20 & 13 & 12 & 4 \\
70 & 21 & 16 & 12 & 11 & 4 \\
100 & 24 & 23 & 15 & 12 & 12 \\
\hline
\end{tabular}

the ideal status of the network, and (iii) one based on random initial solutions. For the latter option, the algorithm was run for 100 different random initial solutions and the best result was kept. Table 4 presents the average percentage difference between the best solutions obtained with the heuristic (considering the three different options for the initial solution) and the optimal value or best lower bound reported by Cplex. That difference is less than $5 \%$ for each combination of number of vertices and budget, and the maximum values are observed for instances with large number of vertices. When only the instances that are solved to optimality are considered, the average gap is $0.39 \%$ and the maximum gap is $3.33 \%$. The average computing times (expressed in seconds) for the heuristic using the three options for the initial solution are shown in Table 5. The running time increases with the size of the instances and is shortest for the instances with the $100 \%$ of budget. Additionally, the computing time required by the heuristic is, on average, only the $4.3 \%$ of that required by Cplex.

Table 4: Average (\%) gap for the best solution found with the KP-based heuristic

\begin{tabular}{cccccc}
\hline & \multicolumn{5}{c}{ Number of vertices } \\
\cline { 2 - 6 } Budget (\%) & 100 & 200 & 400 & 500 & 1000 \\
\hline 20 & 0.30 & 0.77 & 2.46 & 2.81 & 4.90 \\
50 & 0.58 & 1.27 & 2.18 & 2.41 & 3.35 \\
70 & 0.53 & 1.00 & 1.35 & 1.50 & 1.74 \\
100 & 0.13 & 0.21 & 0.32 & 0.41 & 0.53 \\
\hline
\end{tabular}

The results reveal an excellent performance of the KP-based heuristic for finding good feasible solutions for the AAUP in acceptable running times. 
Table 5: Average computing time, expressed in seconds, for the KP-based heuristic

\begin{tabular}{crrrrr}
\hline & \multicolumn{5}{c}{ Number of vertices } \\
\cline { 2 - 6 } Budget (\%) & 100 & \multicolumn{1}{c}{200} & \multicolumn{1}{c}{400} & \multicolumn{1}{c}{500} & 1000 \\
\hline 20 & 27.51 & 32.69 & 80.31 & 87.94 & 102.01 \\
50 & 38.79 & 48.50 & 100.59 & 103.55 & 117.67 \\
70 & 39.85 & 44.35 & 84.35 & 85.53 & 108.23 \\
100 & 27.61 & 34.29 & 60.72 & 64.04 & 95.50 \\
\hline
\end{tabular}

For about $67 \%$ of the instances, the heuristic generates the best results when the initial solution is created based on the ideal status of the network, while, for almost $30 \%$ of the instances, the best solution is found when the algorithm starts from 100 random solutions.

\subsection{Results for the VNS heuristic}

We used a designed experiment both to study the usefulness of the different components of the VNS algorithm and to tune its parameters $\alpha, \beta$ and $\gamma$. According to the results, the two neighbourhoods, the strategic oscillation, and the shaking phase contribute significantly to the performance of the VNS algorithm. It also shows that the parameter setting influence the computing time rather the solution quality.

Based on the experiment results, we set $\alpha=4, \beta=3$, and $\gamma=1$. Finally, we set the stopping criterion for the VNS to 100. In other words, we perform the shaking procedure 100 times.

Table 6 shows, for each combination of budget and number of vertices, the average gap between the best solution found by the VNS algorithm and the optimal solution or best lower bound provided by Cplex. That gap is largest for the instances with budget level $20 \%$ and $50 \%$ and increases with the number of vertices. When only the instances solved to optimality by Cplex are taken into account, the average percentage gap is $0.60 \%$, with a maximum of $3.60 \%$. The average computing times are presented in Table 7 . The computing times increase with both the number of vertices and the budget level, but the impact of the number of vertices is substantially larger than the impact of the budget level. 
Table 6: Average \% gap for the best feasible solutions obtained with the VNS heuristic

\begin{tabular}{cccccc}
\hline & \multicolumn{5}{c}{ Number of vertices } \\
\cline { 2 - 6 } Budget (\%) & 100 & 200 & 400 & 500 & 1000 \\
\hline 20 & 0.80 & 1.46 & 2.85 & 2.62 & 5.74 \\
50 & 0.84 & 1.66 & 2.78 & 2.42 & 4.65 \\
70 & 0.77 & 1.41 & 2.03 & 1.88 & 2.98 \\
100 & 0.09 & 0.26 & 0.45 & 0.43 & 0.77 \\
\hline
\end{tabular}

Table 7: Computing time (s) for the VNS heuristic

\begin{tabular}{cccccc}
\hline & \multicolumn{5}{c}{ Number of vertices } \\
\cline { 2 - 6 } Budget (\%) & 100 & 200 & 400 & 500 & 1000 \\
\hline 20 & 1.47 & 8.82 & 74.58 & 127.45 & 954.99 \\
50 & 2.06 & 11.38 & 95.11 & 166.40 & 1178.07 \\
70 & 2.41 & 13.51 & 104.32 & 180.84 & 1283.43 \\
100 & 2.90 & 17.97 & 142.06 & 241.99 & 1497.67 \\
\hline
\end{tabular}

\subsection{Comparison of the two heuristics}

In order to compare the two different heuristic approaches, we present in Table 8, for each combination of budget and number of vertices, the average percentage difference between the best solution found by the VNS algorithm and the best solution generated by the KP-based heuristic. On average, the KP-based heuristic generates better solutions, particularly for the instances with a tight budget and a large number of vertices. Additionally, while, for the small-sized instances, the computing times for the KP-based heuristic are on average greater than those required by the VNS, for the instances with 1000 vertices, the computing time of the former is on average only $0.17 \%$ of the computing time required by the latter.

\section{$7 \quad$ Potential practical impact}

After having verified the excellent performance of the two heuristic approaches to tackle the AAUP, we would like to highlight their potential for solving real-life decision problems. A a matter of fact, large improvements in accessibility can be obtained by allocating scarce resources properly. To 
Table 8: Average \% difference between the best solutions generated with the VNS approach and the KP-based heuristic

\begin{tabular}{crrrrr}
\hline & \multicolumn{5}{c}{ Number of vertices } \\
\cline { 2 - 6 } Budget $(\%)$ & 100 & 200 & 400 & 500 & 1000 \\
\hline 20 & 0.49 & 0.68 & 0.37 & 0.37 & 0.77 \\
50 & 0.27 & 0.39 & 0.58 & 0.60 & 1.22 \\
70 & 0.24 & 0.40 & 0.67 & 0.68 & 1.21 \\
100 & -0.04 & 0.05 & 0.13 & 0.02 & 0.24 \\
\hline
\end{tabular}

show how the algorithms we have discussed contributed to this end, we measure for each experiment the improvement in accessibility. First, we determine the maximum possible improvement as the difference between the measure of accessibility for the network at its present status and the measure of accessibility for the improved network ignoring the budget constraint. That estimate represents the target improvement for each instance. Once the target has been fixed, we calculate the percentage improvement that is achieved for each given budget level. Table 9 presents the average percentage improvement in accessibility for each combination of budget level and numbers of vertices. From this table, it can be observed that, on average, approximately $70 \%$ and $90 \%$ of the improvement target is obtained when the budget level is set to $20 \%$ and $50 \%$ of the total budget, respectively. Note that the improvement target is not achieved with $100 \%$ the total budget. This is due to the fact that the target was computed ignoring the budget constraint.

Table 9: Percentage of accessibility improvement achieved

\begin{tabular}{cccccc}
\hline & \multicolumn{5}{c}{ Number of vertices } \\
\cline { 2 - 6 } Budget (\%) & 100 & 200 & 400 & 500 & 1000 \\
\hline 20 & 69.52 & 70.66 & 71.66 & 72.58 & 70.80 \\
50 & 90.01 & 90.29 & 90.41 & 90.89 & 90.39 \\
70 & 95.85 & 95.57 & 95.68 & 95.88 & 95.91 \\
100 & 99.29 & 98.99 & 98.97 & 99.01 & 99.00 \\
\hline
\end{tabular}




\section{Conclusions}

We have defined the accessibility arc upgrading problem (AAUP), a network upgrading problem for which real-life applications can be found in several domains such as transportation, telecommunications and logistics. The problem was formulated as an special case of the budget constrained minimum cost flow problem (BC-MCFP).

An analytical study of some special cases of the AAUP provided some theoretical results on the complexity of the problem. Furthermore, based on the insights obtained from the analytical study, we generated a solution approach (which we called the knapsack problem-based heuristic) that performs excellently in terms of efficiency and solution quality. Although this approach might lack the flexibility to deal with additional constraints, it could easily act as a building block for an algorithm designed for more complicated problems.

We also proposed a second solution approach for the AAUP, namely a variable neighbourhood search with strategic oscillation. The VNS algorithm exploits the underlying network flow structure of the problem when defining and evaluating the neighbourhoods. At each iteration, the VNS algorithm considers a feasible upgrading strategy that can be evaluated by solving a minimum cost flow problem. Therefore, by properly defining a function that evaluates the feasibility of a solution, this approach can be easily extended to situations in which additional constraints have to be considered. The experiments illustrate the good performance of our VNS algorithm.

Finally, we pointed out the potential of this study for real-life decision processes. It was shown how the algorithms we have proposed lead to large improvements in accessibility by allocating scarce resources properly.

\section{References}

A. Antunes, A. Seco, and N. Pinto. An accessibility-maximization approach to road network planning. Computer-Aided Civil and Infrastructure Engineering, 18:224-240, 2003.

A.M. Campbell and T.J. Lowe. Upgrading arcs to minimize the maximum travel time in a network. Networks, 47:72-80, 2006. 
Chris Donnges. Improving Access in Rural Areas. Technical report, International Labour Office, Bangkok, 2003.

K.U. Drangmeister, S.O. Krumke, M.V. Marathe, H. Noltemeier, and SS Ravi. Modifying edges of a network to obtain short subgraphs. Theoretical Computer Science, 203:91-121, 1998.

P Hansen and N Mladenovic. Search methodologies. Introductory tutorials in optimization and decision support techniques, chapter Variable neighborhood search, pages 211-238. Springer, 2005.

H Kellerer, U Pferschy, and D Pisinger. Knapsack Problems. Springer Verlag. Springer, 2005.

D Klingman, A Napier, and J Stutz. NETGEN: A Program for Generating Large Scale Capacitated Assignment, Transportation, and Minimum Cost Flow Network Problems. Management Science, 20:814-821, 1974.

S. Krumke, M. Marathe, H. Noltemeier, R. Ravi, and S. Ravi. Approximation algorithms for certain network improvement problems. Journal of Combinatorial Optimization, 2:257-288, 1998.

P. Maya Duque and K. Sörensen. A GRASP metaheuristic to improve accessibility after a disaster. OR Spectrum, pages 1-18, 2011.

L. Murawski and R. Church. Improving accessibility to rural health services: The maximal covering network improvement problem. Socio-Economic Planning Sciences, 43:102-110, 2009.

B. Santos, A. Antunes, and E. Miller. Interurban road network planning model with accessibility and robustness objectives. Transportation Planning and Technology, 33:297-313, 2010.

M. Scaparra and R. Church. A GRASP and path relinking heuristic for rural road network development. Journal of Heuristics, 11:89-108, 2005. 